\title{
UNIVERSAL PACKET ROUTING WITH ARBITRARY BANDWIDTHS AND TRANSIT TIMES
}

\author{
BRITTA PEIS AND ANDREAS WIESE
}

\begin{abstract}
A fundamental problem in communication networks is store-andforward packet routing. In a celebrated paper Leighton, Maggs, and Rao [12] proved that the length of an optimal schedule is linear in the trivial lower bounds congestion and dilation. However, there has been no improvement on the actual bounds in more than 10 years. Also, commonly the problem is studied only in the setting of unit bandwidths and unit transit times. In this paper, we prove bounds on the length of optimal schedules for packet routing in the setting of arbitrary bandwidths and arbitrary transit times. Our results generalize the existing work to a much broader class of instances and also improve the known bounds significantly. For the case of unit transit times and bandwidths, we improve the best known bound of $39(C+D)$ to $23.4(C+D)$, where $C$ and $D$ denote the congestion and dilation, respectively. If every link in the network has a certain minimum transit time or capacity we improve this bounds to up to $4.25(C+D)$. Key to our results is a framework which employs tight bounds for instances where each packet travels along only a small number of edges. Further insights for such instances would reduce our constants even more.
\end{abstract}

\section{INTRODUCTION}

The problem to transport packets within a communication network on time is one of the most fundamental problems in parallel or distributed systems. Routing protocols need to be designed that find a path for each individual packet along which it is routed through the network. Once the paths are chosen, the protocol needs to determine a schedule which defines when each packet traverses the communication links of its path. In this paper we study universal routing protocols [22] for the latter task. They are universal in the sense that they work for arbitrary networks, as well as for arbitrary paths (e.g., the predefined paths need not necessarly be shortest paths).

Most parallel and distributed systems utilize store-and-forward packet routing in which no two packets can cross the same link simultaneously ("unit bandwith"), and each packet needs one unit of time to traverse a single link ("unit transit times"). Usually, the performance of a routing protocol is measured in terms of the two trivial lower bounds $C$ and $D$. The congestion $C$ denotes the maximal number of paths using a single link. The dilation $D$ denotes the maximal length of a path along which a packet has to be routed.

The break-through result for store-and-forward packet routing is certainly due to Leighton, Maggs and Rao [12] that proves the existence of a routing protocol whose length is linear in $C+D$. However, the hidden constants in their result are very large. Scheideler [22] improves this by showing the existence of a protocol of length $39(C+D)$. 
In contrast to previous work, we provide bounds for the more general setting of store-and-forward packet routing with arbitrary transit times and bandwiths. For the special case of unit transit times and bandwiths, we can even improve the so far best known result of Scheideler from $39(C+D)$ to $23.4(C+D)$.

Note that the restriction to unit transit times and bandwiths in ordinary storeand-forward packet routing is of course justifyable as one can always transform an instance with arbitrary transit times and bandwiths to the latter by splitting the edges and adding parallel edges, respectively. However, as we will show in this paper, the bounds on the length of an optimal protocol improve considerably for increasing minimum bandwith or increasing minimum transit times (or both) of the communication links, see Table 1. For example, if we know that the bandwith is at least two on each link, the bound decreases already to $20.52(C+D)$, even if we have unit transit times. Note that in the case of arbitrary bandwidths the (generalized) congestion $C$ depends on the bandwidths of the edges. In particular, the improvement of the bound is not a trivial consequence of the increased bandwidth.

1.1. The model. We model the communication network by a directed graph $G=$ $(V, E)$ whose edges correspond to the links of the network. Each edge $e \in E$ is equipped with a certain bandwidth $b_{e} \in \mathbb{N}$ denoting the maximal number of packets that are allowed to enter $e$ simultaneously, and a certain transit time $\tau_{e} \in \mathbb{N}$ denoting the time needed for a single packet to traverse $e$. We define

$$
b:=\min _{e \in E} b_{e} \quad \text { and } \quad \tau:=\min _{e \in E} \tau_{e} .
$$

Each packet $M_{i}$ must be sent through the network from its origin $s_{i} \in V$ to its destination $t_{i} \in V$ along a predefined $s_{i}$ - $t_{i}$-path $P_{i}$. Thus, each packet consists of a tripel $M_{i}=\left(s_{i}, t_{i}, P_{i}\right)$. We let $\mathcal{M}=\left\{M_{1}, M_{2}, M_{3}, \ldots, M_{|\mathcal{M}|}\right\}$ denote the set of all packets that have to be sent through the network. We assume that time is discrete and that all packets take their steps simultaneously.

1.1.1. The packet routing problem. A routing protocol, or feasible packet routing schedule for the instance $I=(G, \mathcal{M})$ is now a schedule which defines what packets enter what edges at what times (respecting transit times, bandwiths and predefined paths). The corresponding packet routing problem is to minimize the makespan of such a schedule, which is the last point in time when a packet reaches its destination vertex.

1.1.2. Dilation and congestion. The two trivial lower bounds dilation and congestion carry easily over from unit to more general transit times and bandwidths. For each packet $M_{i}$ we define $D_{i}$ to be the length of $P_{i}$ and $D$ to be the maximal length of a path, i.e.,

$$
D_{i}:=\sum_{e \in E: e \in P_{i}} \tau_{e} \text { and } D:=\max _{i \in N} D_{i} .
$$

Also, for each edge $e \in E$ we define $C_{e}$ to be the number of paths using $e$. We define the congestion $C$ by

$$
C:=\max _{e \in E}\left\{\left\lceil C_{e} / b_{e}\right\rceil\right\} .
$$

Clearly, the dilation $D$ as well as the congestion $C$ provide lower bounds on the length of an optimal schedule. 


\begin{tabular}{|c|c|c|c|c|c|c|}
\hline \multicolumn{7}{|c|}{ bound on schedule length } \\
\hline & $\tau=1$ & $\tau=2$ & $\tau=5$ & $\tau=10$ & $\ldots$ & $\tau=63$ \\
\hline \hline$b=1$ & $23.40(C+D)$ & $23.21(C+D)$ & $18.55(C+D)$ & $16.16(C+D)$ & $\ldots$ & $4.25(C+D)$ \\
\hline$b=2$ & $20.52(C+D)$ & $18.13(C+D)$ & $15.58(C+D)$ & $14.06(C+D)$ & $\ldots$ & $4.25(C+D)$ \\
\hline$b=5$ & $16.20(C+D)$ & $14.51(C+D)$ & $12.98(C+D)$ & $12.17(C+D)$ & $\ldots$ & $4.25(C+D)$ \\
\hline$b=10$ & $14.04(C+D)$ & $12.33(C+D)$ & $11.87(C+D)$ & $11.33(C+D)$ & $\ldots$ & $4.25(C+D)$ \\
\hline$\ldots$ & $\ldots$ & $\ldots$ & $\ldots$ & $\ldots$ & $\ldots$ & $\ldots$ \\
\hline$b \rightarrow \infty$ & $7.46(C+D)$ & $7.46(C+D)$ & $7.46(C+D)$ & $7.46(C+D)$ & $\ldots$ & $4.25(C+D)$ \\
\hline \multicolumn{7}{|c|}{ TABLE 1. Bounds for schedules depending on $b$ and $\tau$ obtained in }
\end{tabular}

TABLE 1. Bounds for schedules depending on $b$ and $\tau$ obtained in this paper. Note that for technical reasons which will become clear in Section 4 the bounds do not improve when choosing $\tau$ larger than 63 .

1.1.3. Remark regarding large $(C+D)$. In our analysis we will always assume that $C+D$ is large enough such that $\lceil k \cdot(C+D)\rceil \approx k \cdot(C+D)$ for certain constants $k$. This simplifies the calculations and was also implicitly used by Scheideler [22]. In order to give a fair comparison with his bounds we use this assumption as well. Moreover, we believe that also for instances where $C+D$ is small, our techniques can be used to prove good bounds for the optimal makespan. However, this would require further case distinctions which is beyond the scope of this paper.

1.2. Our Contribution. We prove bounds for the length of an optimal packet routing schedule in the case of arbitrary transit times and bandwidths for the edges. For the classical setting where $b=1$ and $\tau=1$ we improve the best known bound of $39(C+D)$ due to Scheideler [22] to $23.4(C+D)$. Even more, for increasing $b$ or $\tau$ our bounds even improve even further to up to 7.46(C+D) and $4.25(C+D)$, respectively. See Table 1 for an overview for some values of $b$ and $\tau$.

The key insight for our analysis is to prove and exploit a good bound for schedules with very small dilation: We show that if $D \leq \tau+1$, then there is always a schedule of length $C+D-1$. Note that this bound is tight, since there exist instances which require a schedule of this length (e.g., consider $C$ packets that need to take the same path of length $D$ ). Our proof framework uses this insight in order to develop good bounds for general instances.

Also, our approach points into a direction of promising further research: If one could prove similarly tight bounds for instances with, e.g., $D \leq k \tau+1$ for small $k$, our proof framework would immediately give even better bounds for all instances.

This paper consists of two parts: In Section 2 we prove a bound of $C+D-1$ for instances with $D \leq \tau+1$. Then, in Section 3, we prove our bounds for general instances, using the insights obtained in Section 2.

1.3. Related Work. The packet routing problem and related problems are widely studied in the literature.

As mentioned above, Leighton et al. show that there is always a routing schedule that finishes in $O(C+D)$ steps [12]. In [13], Leighton et al. present an algorithm that finds such a schedule in polynomial time. However, this algorithm is not suitable for practical applications since the hidden constants in the schedule length are very large. 
There are various "constant factor approximation"-results in the area of packet routing which are based on the fact that a constant factor approximation on an optimal schedule for store-and-forward packet routing exists. Our improved bounds thus have implications for all of these results. We mention some examples, where store-and-forward packet routing is a subproblem that needs to be solved:

Srinivasan and Teo [23] present a constant factor approximation algorithm for packet routing with variable paths, i.e., in the setting where the routing paths are not part of the input, but need to be found by the algorithm. Koch et al. [11] improve and generalize this algorithm to the more general message routing problem (where each message consists of several packets). In both results, suitable paths are found that yield a constant factor approximation on the minimum of $C+D$ over all possible choices of paths. The remaining problem is then the ordinary packet routing problem. For the case that each vertex of a grid graph is the start vertex of at most one packet, Mansour and Patt-Shamir [16] prove the existence of a constant factor approximation on an optimal schedule by reducing the problem to an ordinary packet routing problem.

There are other result that go in the direction of the work of Leighton et al. [12]. E.g., Meyer auf der Heide et al. [9] present a randomized online-routing protocol which finishes after at most $O(C+D+\log N)$ steps if all paths are shortest paths. Rabani et al. [20] give a distributed algorithm which guarantees a bound of $O(C)+$ $\left(\log ^{*} n\right)^{O\left(\log ^{*} n\right)} D+\operatorname{poly}(\log n)$. This is improved by Ostrovsky et al. [17] who give a local protocol that needs at most $O\left(C+D+\log ^{1+\epsilon} N\right)$ steps.

Packet routing is also studied in the case of special graph topologies. Leung et al. [15, chapter 37] study packet routing on certain tree topologies. Leighton, Makedon and Tollis [14] show that the permutation routing problem on an $n \times n$ grid can be solved in $2 n-2$ steps using constant size queues. Rajasekaran [21] presents several randomized algorithms for packet routing on grids.

Studying the complexity for the ordinary packet routing problem, Di Ianni shows that the delay routing problem [4] is $N P$-hard. The proof implies that the packet routing problem on general graphs is $N P$-hard as well. In [18], the authors improve this by giving non-approximability results for the packet routing problem on several graph classes as well as algorithms for the problem on trees. The same authors present $N P$-hardness results and algorithms for some cases of the packet routing problem on grid graphs [19].

Busch et al. [3] study the direct routing problem, that is the problem of finding a routing schedule such that a packet is never delayed once it has left its start vertex. They give complexity results and algorithms for finding direct schedules. Finally, Adler et al. [1, 2] study the problem of scheduling as many packets as possible through a given network in a certain time frame. They give approximation algorithms and $N P$-hardness results.

With our results, we also contribute to the area of multi-commodity flows over time which is widely studied, e.g., see $[5,6,7,8,10]$. The multi-commodity flow over time problem is equivalent to the (classical) packet routing problem with variable paths if we additionally require unit edge capacities, unit transit times, and integral flow values. Thus, packet routing with variable paths and arbitrary transit times and bandwiths corresponds to the integral multi-commodity flow problem over time. 


\section{Tight Bound for Instances with Small Dilation}

Ideally, we would like to determine each value combination of $C, D, b$, and $\tau$ a tight upper bound on the maximal length of an optimal schedule for instances with these parameters. In this section, we make a first step towards this goal: We give a tight bound for instances with $D \leq \tau+1$. As we will see in Section 3, this insight will allow us to prove good upper bounds for all instances.

For the sake of analysis, we replace every edge $e \in E$ with transit time $\tau_{e}$ by a path consisting of $\tau_{e}$ edges, all with the same bandwidth as $e$. In the resulting graph, we call every vertex that was created due to this transformation a small vertex. All other vertex are called big vertices. We say a small path is a path connecting two big vertices. Hence, $\tau$ is the maximum length of a small path. (Note that this assumption makes the problem slightly more general since now packets are allowed to have their start vertex "in the middle" of an edge. We introduce this generalization because it will become handy in our proof framework later.) To simplify notation later we introduce the notion $A^{b, \tau}(C, D)$.

Definition 1. Let $\mathcal{I}^{b, \tau}(C, D)$ be the set of all packet routing instance with minimum bandwidth $b$, minimum transit time $\tau$, congestion $C$, and dilation $D$. We define

$$
A^{b, \tau}(C, D):=\max _{I \in \mathcal{I}^{b, \tau}(C, D)} O P T(I),
$$

where $O P T(I)$ denotes the length of an optimal schedule for an instance $I$.

The main result of this section is given in the following theorem.

Theorem 2. Let I be an instance of the packet routing problem with $D \leq \tau+1$. Then there is a schedule for I whose makespan is bounded by $C+D-1$. Moreover, $A^{b, \tau}(C, D)=C+D-1$ if $D \leq \tau+1$.

2.1. Proof of Theorem 2. The strategy of the proof is the following: We assume that we are given an instance $I$ of the packet routing problem with $D \leq \tau+1$. Due to the latter assumption the path of each packet uses at most two small paths. Thus, we can divide the packets using any small path $P$ into two sets $\mathcal{M}_{P}^{1}$ and $\mathcal{M}_{P}^{2}$, such that $\mathcal{M}_{P}^{1}$ contains the packets for which $P$ is the first small path, and $\mathcal{M}_{P}^{2}$ contains the packets for which $P$ is the second small path.

In a first step, we transform $I$ into an instance $I^{\prime}$ such that $O P T\left(I^{\prime}\right) \geq O P T(I)$ and for every small path $P^{\prime}$ either $\mathcal{M}_{P^{\prime}}^{1}=\emptyset$ or $\mathcal{M}_{P^{\prime}}^{2}=\emptyset$. This procedure reduces the complexity of the instance significantly. While performing the necessary changes we do not change $C, D$ or $\tau$ at all. It turns out that the resulting instance $I^{\prime}$ is an instance of the packet routing problem whose underlying graph topology is the union of directed spiders.

We show that for each connected component there is a schedule which finishes after at most $C+D-1$ steps. Since $O P T\left(I^{\prime}\right) \leq C+D-1$ and $O P T(I) \leq O P T\left(I^{\prime}\right)$, it follows that

$$
A^{b, \tau}(C, D) \leq C+D-1 \text {. }
$$

It is straight forward to construct instances $I$ with $D \leq \tau+1$ and $O P T(I)=$ $C+D-1$. For example, consider an instance with only one edge which is used by $C \cdot b$ packets. This shows that the bound is tight, i.e.,

$$
A^{b, \tau}(C, D)=C+D-1 \text {. }
$$



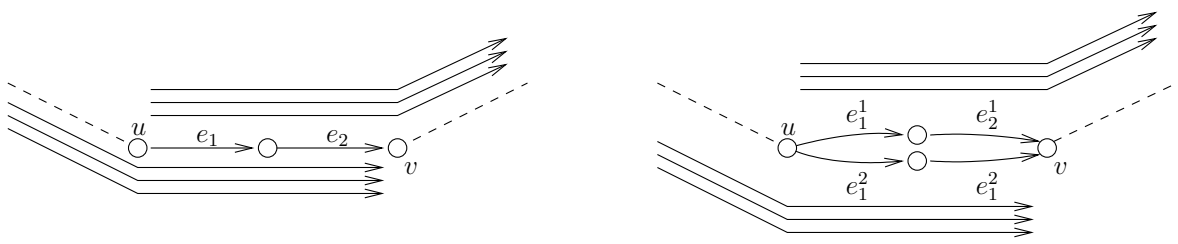

FiguRe 2.1. A sketch of the transformation from $I$ to $I^{\prime}$ with $\tau=2$ and $u$ and $v$ being big vertices. In the left instance $I$ all six paths use $e_{1}$ and $e_{2}$. In the transformed instance $I^{\prime}$ on the right the upper three paths use $e_{1}^{1}$ and $e_{2}^{1}$ since $\left\{e_{1}, e_{2}\right\}$ is their first small path. Similarly, the lower three paths use $e_{1}^{2}$ and $e_{2}^{2}$.

We now describe the transformation from $I$ to $I^{\prime}$ and the proof that $O P T\left(I^{\prime}\right) \leq$ $C+D-1$ in detail.

2.1.1. Transformation from $I$ to $I^{\prime}$. Note that for an optimal schedule for $I$ we can assume w.l.o.g. that on any small path $P$ no packet in $\mathcal{M}_{P}^{2}$ ever delays a packet in $\mathcal{M}_{P}^{1}$. Consider a small path $P=\left\{e_{1}, e_{2}, \ldots, e_{k}\right\}$ connecting two big vertices $u$ and $v$. We substitute the path by two paths $P^{1}=\left\{e_{1}^{1}, e_{2}^{1}, \ldots, e_{k}^{1}\right\}$ and $P^{2}=\left\{e_{1}^{2}, e_{2}^{2}, \ldots, e_{k}^{2}\right\}$ where each edge $e_{j}^{i}$ has the same bandwidth as $e_{j}$. In the new instance $I^{\prime}$, we define that the packets of $\mathcal{M}_{P}^{1}$ use $P^{1}$, whereas the packets in $\mathcal{M}_{P}^{2}$ use $P^{2}$.

Now, let $M \in \mathcal{M}_{P}^{1}$ be a packet that uses the edges $e_{\ell}^{1}, \ldots, e_{\ell^{\prime}}^{1}$ for $1 \leq \ell \leq \ell^{\prime} \leq k$. (As mentioned above, when analyzing the general setting in Section 3, we also need to consider instances in which the start- and destination vertices correspond to small vertices.) For each such packet $M$, we introduce an artificial packet $M^{\prime}$ whose path consists of the edges $e_{\ell}^{2}, \ldots, e_{\ell^{\prime}}^{2}$. If $\ell^{\prime}=k$ we call $M^{\prime}$ a far artifical packet. We do this procedure with every packet in $\mathcal{M}_{P}^{1}$. Denote by $I^{\prime}$ this transformed instance. For every small path $P=\left\{e_{1}, e_{2}, \ldots, e_{k}\right\}$ in $G$ we denote by $\mathcal{M}_{P}^{\prime \prime}$ and $\mathcal{M}_{P}^{\prime 2}$ the packets in $I^{\prime}$ which use edges of $P^{1}=\left\{e_{1}^{1}, e_{2}^{1}, \ldots, e_{k}^{1}\right\}$ and $P^{2}=\left\{e_{1}^{2}, e_{2}^{2}, \ldots, e_{k}^{2}\right\}$, respectively. See Figure 2.1 for a sketch.

2.1.2. Bound for $O P T\left(I^{\prime}\right)$. For packet routing on a path it is optimal to schedule the packets by the farthest destination first rule. Hence, for the instance $I^{\prime}$ there is always an optimal schedule in which no far artificial packet is ever delayed by a non-artificial packet. Thus, our transformation ensures that $O P T\left(I^{\prime}\right) \geq O P T(I)$. Note that in $I^{\prime}$ we still have that $D \leq \tau+1$. Moreover, in $I^{\prime}$ we have that either $\mathcal{M}_{P^{\prime}}^{\prime 1}=\emptyset$ or $\mathcal{M}_{P^{\prime}}^{\prime 2}=\emptyset$ for each small path $P^{\prime}$. Also, the transformation did not change $C, D, b$, or $\tau$. We observe that the topology of $I^{\prime}$ is a directed forest, i.e., the union of directed trees. Even more, in each connected component there is at most one vertex with a larger degree than 2 . We call directed trees with this property directed spiders. We want to prove the following claim.

Claim. There is a schedule for $I^{\prime}$ whose length is at most $C+D-1$.

Take a small path $P^{\prime}$ with bandwidth $b \geq 2$ (recall that all edges on a small path have the same bandwidth). We replace $P^{\prime}$ by $b$ paths $P_{1}^{\prime \prime}, \ldots, P_{b}^{\prime \prime}$ of unit bandwidth. The packets using $P^{\prime}$ in $I^{\prime}$ are now distributed among the new paths such that no path is used by more than $C$ packets. We do this transformation with each 
small path. By construction any feasible schedule of the resulting instance can be transformed to a feasible schedule for $I^{\prime}$ with the same length.

Consider one connected component. It is a directed spider where each edge has unit bandwidth. It was shown in [18] that for instances of the packet routing problem on directed trees there is always a schedule of length $C+D-1$. However, for this special case we give a simpler direct proof.

It is known that any set of paths on a directed tree can be colored with $C$ colors such that no two paths with the same color share an edge (where $C$ denotes the maximal number of paths which share an edge). Such a coloring can be obtained by first reducing the problem on directed star graphs (i.e., directed trees with diameter two) to edge-coloring in bipartite multigraphs. Do this computation for each star. Then, the solutions for the star graphs are glued together to obtain a global solution, see [18] for details. We assume in the sequel that each packet $M_{i}$ is colored with a color $c_{i} \in\{0, \ldots, C-1\}$ such that no two packets with the same color share an edge.

2.1.3. Routing Schedule. Using the coloring defined above, we want to define a schedule which finishes after at most $C+D-1$ steps. We define $v$ to be the single vertex in the component which has a larger degree than two (or an arbitrary vertex if there is no such vertex). For each vertex $v^{\prime}$ we define a value $h\left(v^{\prime}\right)$. If there is a directed path from $v$ to $v^{\prime}$ of length $\ell$ then we define $h\left(v^{\prime}\right):=\ell$. If there is a directed path from $v^{\prime}$ to $v$ of length $\ell$ then we define $h\left(v^{\prime}\right):=-\ell$. Note that since our component is a directed spider one of the two cases must apply.

Now we define our routing schedule. It is a direct schedule, i.e., every packet waits in its start vertex for some time and then proceeds to its destination without any further delay. We assign each packet $M_{i}$ an initial waiting time of $d_{i}:=\left(c_{i}+h\left(s_{i}\right)\right) \bmod C$. Note that this already characterizes the schedule completely.

Lemma 3. The defined schedule is feasible and finishes after at most $C+D-1$ steps. Hence, $O P T\left(I^{\prime}\right) \leq C+D-1$.

Proof. Assume on the contrary that at time $t$ two packets $M_{i}, M_{i^{\prime}}$ collide when leaving some vertex $v^{\prime}$. Hence, $t=d_{i}-h\left(s_{i}\right)+h\left(v^{\prime}\right)=d_{i^{\prime}}-h\left(s_{i^{\prime}}\right)+h\left(v^{\prime}\right)$. This implies that $\left(c_{i}+h\left(s_{i}\right)\right) \bmod C-h_{i}=\left(c_{i^{\prime}}+h\left(s_{i^{\prime}}\right)\right) \bmod C-h_{i^{\prime}}$ and hence $c_{i} \equiv$ $c_{i^{\prime}}$ mod $C$. However, this contradicts that the $c_{i}$ values form a valid coloring.

By definition, each packet waits in its start vertex for at most $C-1$ steps and then moves to its destination. Hence, the length of the schedule is bounded by $C+D-1$. Doing the described adjustments with every connected component shows that $\operatorname{OPT}\left(I^{\prime}\right) \leq C+D-1$.

Using the insights above we obtain the proof for Theorem 2 .

Proof of Theorem 2. Let $I$ be an instance of the packet routing problem. We transform $I$ to $I^{\prime}$ as described above. Since

$$
O P T(I) \leq O P T\left(I^{\prime}\right) \leq C+D-1
$$

it follows that $A^{b, \tau}(C, D) \leq C+D-1$. There are instances where this bound of $C+D-1$ is in fact achieved (see above). Hence the bound is tight and thus $A^{b, \tau}(C, D)=C+D-1$.

Remark: At first glance our technique might seem to work also if $D$ can be as large as $2 \tau$. Unfortunately, this is not the case. Recall that we allow the packets to 
start in the middle of a short path (we will need this property later in our framework for general instances). If there is a packet whose path uses more than $\tau+1$ edges then its path could cross three small paths. Then, our technique to reduce the problem to star graphs does not work any longer. However, if we do not allow the packets to start in the middle of short paths then our bound also holds if we require only that $D \leq 2 \tau$.

\section{High Level Ideas for General Bounds}

In the previous section, we provided a tight bound for $A^{b, \tau}(C, D)$ assuming that $D \leq \tau+1$. Unfortunately, the vast majority of instances does not fall under this category. However, in this section we provide an upper bound for all values $A^{b, \tau}(C, D)$. In order to do this, we provide a framework which uses bounds for instances with small dilation (like $D \leq \tau+1$ ) for proving good bounds for all instances. In particular, we prove the following theorems.

Theorem 4. Let I be an instance of the packet routing problem with minimum bandwidths and transit times $b$ and $\tau$, respectively. There is a feasible schedule for $I$ whose length is bounded by

$$
\left(6.4 \cdot f(\tau+1, b)+2.126 \frac{\tau}{\tau+1}+\delta\right)(C+D)
$$

for a function $f(\tau+1, b)$ which tends to 1 for increasing $\tau$ or $b$ and with $\delta=$ $\frac{1}{60}(\tau+3.01 \cdot f(\tau+1, b)(\tau+1))$.

Note that the value $\delta$ in the theorem above increases for increasing $\tau$. However, if $\tau \geq 63$ the following theorem gives an alternative bound.

Theorem 5. Let I be an instance of the packet routing problem with minimum transit time $\tau \geq 63$. Then there is a feasible schedule for I whose length is bounded by $4.25(C+D)$.

Even more, assuming that one has good upper bounds for instances with small dilation we present a framework which gives good upper bounds for all values $A^{b, \tau}(C, D)$.

Theorem 6. Let $I$ be an instance of the packet routing problem with minimum bandwidths and transit times $b$ and $\tau$, respectively. For any $\ell \in \mathbb{N}$ there is a feasible schedule for I whose length is bounded by

$$
A^{b, \tau}(3.01 \ell \cdot f(\ell, b), \ell) \cdot\left(\frac{2.11}{\ell}+\delta\right)(C+D)
$$

for a function $f(\ell, b)$ which tends to 1 for increasing $\ell$ or $b$ and with $\delta=\frac{1}{60}$.

As an application of this framework, the proof of Theorem 4 uses that $A^{b, \tau}(C, \tau+$ $1)=C+\tau$ as proven in Theorem 2. Also, for the important special case of unit bandwidths and unit transit time (i.e., $b=1$ and $\tau=1$ ) our framework gives the following bound.

Theorem 7. Let $I$ be an instance of the packet routing problem with $b=1$ and $\tau=$ 1. Then there is a feasible schedule for $S$ whose length is bounded by $23.4(C+D)$. 


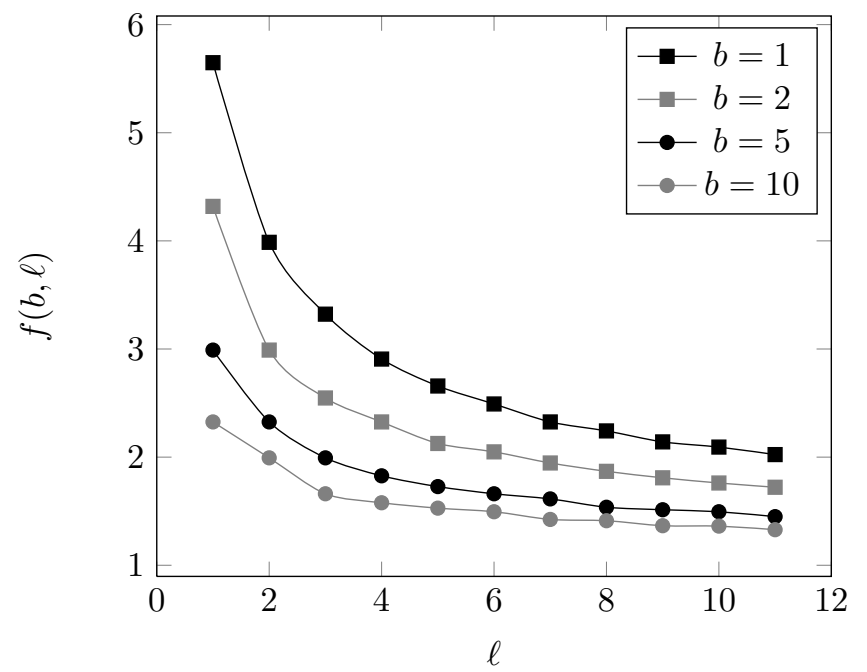

Figure 3.1. The function $f$ for the values of $b$ and $\ell$ which are relevant for the bounds shown in Table 1.

Table 1 shows the bounds obtained by the above theorems for certain values of $b$ and $\tau$. Figure 3.1 shows a plot of the function $f$ used in Theorem 4 and 6 for some values $b$.

In this section we describe the high-level concepts for our proof. The complete proof requires many technical details which we give in Section 4. Our reasoning uses the concepts introduced by Scheideler [22] who proved that there is always a schedule of length $39(C+D)$ for instances with unit transit times and unit capacities.

In the first part of our proof, we give a careful adaption of these concepts to the setting of arbitrary bandwidths and transit times. In the second part of the proof, we introduce our framework. Any good bound for instances with small dilation, e.g., $D \leq \tau+1, D \leq 2 \tau+1$, etc., allows the framework to prove better bounds for general instances (with arbitrary dilation). We incorporate the bounds for instances with $D \leq \tau+1$ (obtained in Section 2) into our framework. In the setting of unit bandwidths and transit times we improve the bound of $39(C+D)$ by Scheideler [22] to $23.4(C+D)$. If even $b \geq 2$ and/or $\tau \geq 2$ we can reduce the constant in front of $(C+D)$ even further.

In the sequel, we will use the concept of infeasible schedules. We call a schedule infeasible if in the schedule some edge $e$ is used by more than $b_{e}$ packets at a time, but the other two properties of feasible schedules are fulfilled. For ease of notation we say a schedule is infeasible if it is not necesarily feasible. We use the following strategy: We start with an infeasible schedule in which no packet is ever delayed. Denote by $S_{0}$ this schedule. We perform the following steps.

- One can enlarge $S_{0}$ by adding a random delay of at most $C / b$ for each packet at the beginning of $S_{0}$, yielding a schedule $S_{1}$. We will show using the Lovász Local Lemma that there are delays for the packets such that $S_{1}$ fulfills certain properties. 
- Inductively, assume that we have an infeasible schedule $S_{i}$ (with $i \geq 1$ ). Using the Local Lemma we show that there are further refinement steps yielding a schedule $S_{i+1}$ which is - intuitively speaking - "more feasible" than $S_{i}$.

- Eventually, we prove that there is a schedule $S_{k}$ with the property that in every interval of length 64 at most $192.1 b$ packets use each edge. Furthermore, we prove that the length of $S_{k}$ is bounded by $1.0626(C+D)$.

Starting with the infeasible schedule $S_{k}$, we establish our framework. Let $\ell \in \mathbb{N}$. Using the Lovász Local Lemma we show that there is an infeasible schedule $S_{k+1}$ that can be partitioned such that in any time-interval of length $\ell$ at most $C_{b_{e}}^{\ell}$ packets traverse each edge $e$. (For a constant $C_{b_{e}}^{\ell}$ to be defined later.) Hence, we can turn $S_{k+1}$ into a feasible schedule by refining each interval of length $\ell$ separately. In order to do this, we treat each of these intervals as a subinstance of the packet routing problem with dilation $\ell$ and congestion $\max _{e}\left\{C_{b_{e}}^{\ell} / b_{e}\right\}$. Hence, it suffices to have good bounds for instances with dilation $D=\ell$ in order to obtain a bound for the original instance. We use our framework with $\ell:=\tau+1$ since Theorem 2 gives a bound for instances with $D \leq \tau+1$. Using the framework one could obtain even better bounds for general instances if one had good upper bounds for instances with slightly higher dilation, e.g., $D \leq k \tau+1$ for some small value $k$. In particular, the larger we can choose $\ell$, the better become our bounds. This can be seen in Table 1 since for increasing $\tau$ the bounds improve. Also, if $b$ increases the bounds improve as well. The reason is that $C_{b}^{\ell} / b$ decreases when $b$ increases. Hence, the congestion in the subinstances (given as $\max _{e}\left\{C_{b_{e}}^{\ell} / b_{e}\right\}$ above) will be smaller for larger values of $b$.

In the following section we give a detailed technical analysis of the reasoning described above.

\section{Technical Analysis}

In this section we give the full proofs of the theorems and techniques stated in Section 3. First, we adapt the concepts of Scheideler [22] to the setting of arbitrary bandwidths and transit times. Then, we introduce our framework which then allows us to prove our bounds for the lengths of optimal schedules.

Let $I$ be an instance of the packet routing problem with congestion $C$ and dilation $D$. Assume that each edge has a bandwidth of at least $b$ and each a transit time of at least $\tau$. Our bounds depend on these four parameters. In particular, they improve if $b$ and $\tau$ increase. As already mentioned in the Introduction, we replace every edge $e$ with transit time $\tau_{e}$ by a path consisting of $\tau_{e}$ edges.

First, we prove the existence of the schedule $S_{k}$ with the property that in every interval of length 64 at most $192.1 b$ packets use each edge. We bound the length of $S_{k}$ later. We define $I_{0}:=\max \{C, D\}$. Let $k:=\left(\log ^{*} I_{0}\right)-1^{1}$. We set $I_{k}:=4$ and

$$
I_{j}:=2^{I_{j+1}}
$$

for all $j$ with $1 \leq j \leq k-1$. Note that $2^{I_{1}} \geq I_{0}$. If $I_{0} \leq 64$ then we define $S_{k}:=S_{0}$. Hence, for the remaining reasoning for the construction of $S_{k}$ we assume that $I_{0}>64$. Let $S_{0}$ be the infeasible schedule in which no packet is ever delayed. We define $D_{0}:=D$. We will prove the existence of schedules $S_{i}$ with certain

\footnotetext{
${ }^{1}$ For our purposes we define $\log ^{*} I_{0}$ to be the smallest integer $k$ such that we need to apply the log-function $k$ times to $I_{0}$ in order to obtain a value of at most 4 .
} 
properties (with $i \geq 1$ ). We denote by $D_{i}$ the length of $S_{i}$. Let $C_{i}$ be the maximum number of packets that use an edge in each interval of length $I_{i}^{3}$ in the schedule $S_{i}$.

We start with the schedule $S_{0}$. We assign each packet an initial random delay. Using the Lovász Local Lemma we prove that there are random delays such that the resulting schedule is "relatively feasible". The schedule $S_{1}$ is the schedule resulting from those "good" initial delays.

Lemma 8. There is an infeasible schedule $S_{1}$ with the property that in every interval of length $I_{1}^{3}$ at most $C_{1} b_{e}$ packets use each edge e with $C_{1}:=\left(1+\frac{3}{I_{1}}\right) I_{1}^{3}$. Also, $D_{1} \leq D+C$.

Proof. We prove the existence of $S_{1}$ using the Lovász Local Lemma. We change $S_{0}$ by giving each packet an initial delay chosen uniformly at random from the set $\{0, \ldots, C-1\}$. This results in an infeasible schedule $S_{1}$ whose length is bounded by $(D+C)$. In order to make $S_{1}$ "relatively feasible" we want to ensure that in $S_{1}$ at most $C_{1} b_{e}$ packets traverse every edge $e$ during any interval of length $I_{1}^{3}$. We ensure this property by using the Lovász Local Lemma. For being able to employ the lemma, we define a bad event $E_{e}$ for each edge $e$. The bad event $E_{e}$ is that there is an interval of length $I_{1}^{3}$ in which more than $C_{1} b_{e}$ traverse $e$. Using the Lovász Local Lemma we show that there are initial delays for the packets such that no bad event occurs. Consider an edge $e$ and an interval $J$ of length $I_{1}^{3}$. Assume that the packets $M_{1}, \ldots, M_{\ell}$ use $e$ (note that $\ell \leq C b_{e}$ ). Let $X_{i}$ be a binary random variable such that $X_{i}=1$ if and only if $M_{i}$ traverses $e$ during $J$. We define $X:=\sum_{i=1}^{\ell} X_{i}$. The initial delays were chosen uniformly at random and hence $\operatorname{Pr}\left[X_{i}=1\right] \leq I_{1}^{3} / C$ for each $i$. This implies $\mathbb{E}[X]=\sum_{i=1}^{\ell} \mathbb{E}\left[X_{i}\right] \leq I_{1}^{3} b_{e}$. Using the Chernoff bounds we derive with $\epsilon=\frac{3}{I_{1}}$ that

$$
\begin{aligned}
\operatorname{Pr}\left[X \geq(1+\epsilon) I_{1}^{3} b_{e}\right] & =\operatorname{Pr}\left[X \geq\left(1+\frac{3}{I_{1}}\right) I_{1}^{3} b_{e}\right] \\
& \leq \exp \left(-9 b_{e} I_{1} / 2\right) .
\end{aligned}
$$

(As usual, $\exp (x):=e^{x}$.) Since there are at most $(D+C)$ intervals of length $I_{1}^{3}$, the probability for $E_{e}$ is bounded by

$$
\operatorname{Pr}\left[E_{e}\right] \leq(D+C) \cdot \exp \left(-9 b_{e} I_{1} / 2\right) .
$$

The dependence of each bad event $E_{e}$ is at most $C D b_{e}-1$. In other words: $E_{e}$ is independent of all but at most $C D b_{e}-1$ other bad events. Hence, in order to employ the Lovász Local Lemma we have to ensure that

$$
\operatorname{Pr}\left[E_{e}\right] \cdot e \cdot C D b_{e}<1
$$

Due to our bound for $\operatorname{Pr}\left[E_{e}\right]$ above it suffices to ensure that

$$
(D+C) \cdot \exp \left(-9 b_{e} I_{1} / 2\right) \cdot e \cdot C D b_{e}<1 .
$$

We show the inequality for $b_{e}=1$ (since then by monotonicity it holds for all $\left.b_{e}\right)$. Also, since $I_{1}$ depends only on $\max \{C, D\}$ it suffices to consider the case that $C=D$. This yields the inequality

$$
2 D \cdot \exp \left(-9 I_{1} / 2\right) \cdot e \cdot D^{2}<1 .
$$

Since we assumed that $I_{0} \leq 2^{I_{1}}$ and hence $D \leq 2^{I_{1}}$ it suffices to ensure that 


$$
2 \cdot 2^{I_{1}} \cdot \exp \left(-9 I_{1} / 2\right) \cdot 2^{2 I_{1}}<1
$$

Since $I_{0}>64$ we have in particular that $I_{1} \geq 4$. Inequality 4.1 holds for $I_{1}=4$ and due to monotonicity also for all $I_{1} \geq 4$. Concluding the lemma we obtained the infeasible schedule $S_{1}$ with the property that in each time interval of length $I_{1}^{3}$ each edge is used by at most $C_{1} b_{e}$ packets.

Denote by $S_{1}$ the schedule whose existence was proved in Lemma 8.Given an infeasible schedule $S_{i}$ we want to prove the existence of a schedule $S_{i+1}$ which is - intuitively speaking - "more feasible" than $S_{i}$. This is again done by giving each packet random delays. We use the Lovász Local Lemma to ensure that there are delays for the packets such that in any interval of length $I_{i+1}^{3}$ only a bounded number of packets use each edge.

Lemma 9. Let $S_{i}$ be an infeasible schedule of length $D_{i}$ and with the property that in every interval of length $I_{i}^{3}$ at most $C_{i} b_{e}$ packets use each edge e for some value $C_{i} \geq I_{i}^{3}$. Then there is an infeasible schedule $S_{i+1}$ with the property that in every interval of length $I_{i+1}^{3}$ at most $C_{i+1} b_{e}$ packets use each edge e, with

$$
C_{i+1}:=C_{i} \cdot\left(1+\frac{5}{I_{i+1}}\right) \cdot \frac{I_{i+1}^{3}}{I_{i}^{3}-I_{i+1}^{3}} .
$$

Moreover, $D_{i+1} \leq\left(1+\frac{1}{I_{i}}\right) D_{i}$ and $C_{i+1} \geq I_{i+1}^{3}$.

Proof. We split the timeline into intervals of length $I_{i}^{4}$. We refine the infeasible schedule $S_{i}$ by enlarging each of these intervals. The schedule $S_{i+1}$ is the concatenation of all enlarged intervals.

Let $J^{i}$ be a time interval of length $I_{i}^{4}$. At the beginning of $J^{i}$ we define for each packet a delay chosen uniformly at random from the set $\left\{0,1, \ldots, I_{i}^{3}-I_{i+1}^{3}-1\right\}$. Denote by $\bar{J}^{i}$ the resulting (enlarged) interval. Note that the length of $\bar{J}^{i}$ is bounded by $I_{i}^{4}+I_{i}^{3}-I_{i+1}^{3}$. We want to ensure that in the resulting schedule within each interval of length $I_{i+1}^{3}$ at most $C_{i+1} b_{e}$ packets use each edge $e$. Using the Lovász Local Lemma we show that there are random delays for the packets such that this property is fulfilled.

Let $e$ be an edge. We define that the bad event $E_{e}^{i+1}$ occurs if there is a subinterval of $\bar{J}^{i}$ with length $I_{i+1}^{3}$ in which more than $C_{i+1} b_{e}$ packets use $e$. Let $J^{\prime}=\left[x, x+I_{i+1}^{3}-1\right] \cap \bar{J}^{i}$ for some $x \in \bar{J}^{i}$. If after the random experiment a packet $M$ uses $e$ during $J^{\prime}$ then in $S_{i}$ it must have used $e$ during the interval $J^{\prime \prime}=\left[x-I_{i}^{3}+I_{i+1}^{3}, x+I_{i+1}^{3}-1\right]$. Since $J^{\prime \prime}$ has length $I_{i}^{3}$ there can be at most $C_{i} b_{e}$ such packets. Denote them by $M_{1}, \ldots, M_{\ell}$. Let $X_{i}$ be a random variable such that $X_{i}=1$ if and only if $M_{i}$ uses $e$ during $J^{\prime}$. We define $X:=\sum_{i=1}^{\ell} X_{i}$. For each $X_{i}$ we get $\operatorname{Pr}\left[X_{i}\right] \leq I_{i+1}^{3} /\left(I_{i}^{3}-I_{i+1}^{3}\right)$. We obtain

$$
\mathbb{E}[X]=\sum_{i=1}^{\ell} \mathbb{E}\left[X_{i}\right] \leq C_{i} b_{e} \cdot \frac{I_{i+1}^{3}}{I_{i}^{3}-I_{i+1}^{3}} .
$$


The Chernoff bounds give that

$$
\begin{aligned}
\operatorname{Pr}\left[X \geq C_{i+1} b_{e}\right] & =\operatorname{Pr}\left[X \geq C_{i}\left(1+\frac{5}{I_{i+1}}\right) \frac{I_{i+1}^{3}}{I_{i}^{3}-I_{i+1}^{3}} b_{e}\right] \\
& \leq \exp \left(-\frac{1}{2}\left(\frac{5}{I_{i+1}}\right)^{2} \cdot C_{i} \cdot \frac{I_{i+1}^{3}}{I_{i}^{3}-I_{i+1}^{3}} b_{e}\right) .
\end{aligned}
$$

Since there are at most $I_{i}^{4}+I_{i}^{3}$ intervals of length $I_{i+1}^{3}$ (like the interval $J^{\prime}$ ) which we need to consider, we obtain

$$
\operatorname{Pr}\left[E_{e}\right] \leq\left(I_{i}^{4}+I_{i}^{3}\right) \exp \left(-\frac{1}{2}\left(\frac{5}{I_{i+1}}\right)^{2} \cdot C_{i} \cdot \frac{I_{i+1}^{3}}{I_{i}^{3}-I_{i+1}^{3}} b_{e}\right) .
$$

The interval $J^{i}$ is the union of $I_{i}$ intervals of length $I_{i}^{3}$. Therefore, by assumption during $J^{i}$ (and by construction also during $\bar{J}^{i}$ ) at most $I_{i} \cdot C_{i} b_{e}$ packets pass $e$. Hence, the dependence of $E_{e}$ is bounded by $I_{i}^{5} \cdot C_{i} b_{e}-1$. Therefore, in order to employ the Lovász Local Lemma, we need to guarantee that

$$
\operatorname{Pr}\left[E_{e}\right] \cdot e \cdot I_{i}^{5} \cdot C_{i} b_{e}<1
$$

In order to analyze whether the inequality holds we study the function

$$
g\left(I_{i}, C_{i}\right)=\left(I_{i}^{4}+I_{i}^{3}\right) \exp \left(-\frac{1}{2}\left(\frac{5}{\log I_{i}}\right)^{2} \cdot C_{i} \cdot \frac{\log ^{3} I_{i}}{I_{i}^{3}-\log ^{3} I_{i}} b_{e}\right) e I_{i}^{5} \cdot C_{i} b_{e} .
$$

We need to show that $g\left(I_{i}, C_{i}\right)<1$. First, we calculate that $\frac{d g}{d C_{i}}<0$ and hence it suffices to ensure that $g\left(I_{i}, I_{i}^{3}\right)<1$ (recall that $C_{i} \geq I_{i}^{3}$ ). Similarly, it suffices to show the bound for $b_{e}=1$. We define $h\left(I_{i}\right):=g\left(I_{i}, I_{i}^{3}\right)$ and set $b_{e}:=1$ which yields

$$
\begin{aligned}
h\left(I_{i}\right) & =\left(I_{i}^{4}+I_{i}^{3}\right) \exp \left(-\frac{1}{2}\left(\frac{5}{\log I_{i}}\right)^{2} \cdot I_{i}^{3} \cdot \frac{\log ^{3} I_{i}}{I_{i}^{3}-\log ^{3} I_{i}}\right) e I_{i}^{5} \cdot I_{i}^{3} \\
& \leq\left(I_{i}^{4}+I_{i}^{3}\right) \cdot I_{i}^{8} \cdot e \cdot \exp \left(-12.5 \cdot \log I_{i}\right) \\
& =: \hat{h}\left(I_{i}\right) .
\end{aligned}
$$

For $I_{i}=16$ calculations show that $\hat{h}\left(I_{i}\right)<1$ and $\frac{d \hat{h}}{d I_{i}}<0$. Hence, if $I_{i} \geq 16$ then there are random delays for the packets such that in each interval $J^{\prime} \subseteq \bar{J}^{i}$ of length at most $I_{i+1}^{3}$ there can be at most $C_{i+1} b_{e}$ packets using any edge $e$.

It remains to show that $C_{i+1} \geq I_{i+1}^{3}$. By assumption $C_{i} \geq I_{i}^{3}$. We calculate that

$$
\begin{aligned}
C_{i+1} & =C_{i} \cdot\left(1+\frac{5}{I_{i+1}}\right) \cdot \frac{I_{i+1}^{3}}{I_{i}^{3}-I_{i+1}^{3}} \\
& \geq I_{1}^{3} \cdot\left(1+\frac{5}{I_{i+1}}\right) \cdot \frac{I_{i+1}^{3}}{I_{i}^{3}-I_{i+1}^{3}} \\
& =\frac{1}{1-\frac{I_{i+1}^{3}}{I_{i}^{3}}} \cdot\left(1+\frac{5}{I_{i+1}}\right) \cdot I_{i+1}^{3} \\
& \geq I_{i+1}^{3} \cdot
\end{aligned}
$$


We apply Lemma 9 iteratively until we have proven the existence of the schedule $S_{k}$ with the respective properties. In particular, since $I_{k}=4$ Lemma 9 shows that in $S_{k}$ in every interval of length $4^{3}=64$ every edge is used by at most $C_{k}$ packets. In the following two lemmata we bound $C_{k}$ and $D_{k}$.

Lemma 10. It holds that $D_{k}<1.0626(D+C)$.

Proof. Due to our refinement steps from Lemmata 8 and 9 we have that $D_{k} \leq$ $(D+C) \prod_{i=1}^{k-1}\left(1+\frac{1}{I_{i}}\right)$. For bounding the latter term we define a sequence $a$. Let $a_{0}:=4, a_{1}:=16, a_{2}:=65536=2^{16}$, and $a_{3}:=2^{65536}$. For $i \geq 4$ we define $a_{i+1}:=2 a_{i}$. Our definition of the $a_{i}$ implies that $\prod_{i=1}^{\infty}\left(1+\frac{1}{I_{i}}\right) \leq \prod_{i=1}^{\infty}\left(1+\frac{1}{a_{i}}\right)$. Therefore, it suffices to show that $\prod_{i=1}^{\infty}\left(1+\frac{1}{a_{i}}\right)<1.0625$. With the definition of the sequence $a$ we are able to use the geometric series for bounding $\sum_{i=3}^{\infty} \frac{1}{a_{i}}$. Since the latter term is very small, defining $a_{i+1}:=2 a_{i}$ does not introduce too much inaccuracy. Let $\alpha:=\prod_{i=3}^{\infty}\left(1+\frac{1}{a_{i}}\right)$. We calculate that

$$
\begin{aligned}
\log (\alpha) & =\sum_{i=3}^{\infty} \log \left(1+\frac{1}{a_{i}}\right) \\
& \leq \sum_{i=3}^{\infty} \frac{1}{a_{i}} \\
& \leq 2^{-32768} .
\end{aligned}
$$

This implies that $\alpha \leq 2^{2^{-32768}}$. Calculations show that

$$
\prod_{i=1}^{\infty}\left(1+\frac{1}{a_{i}}\right) \leq \alpha \cdot\left(1+\frac{1}{a_{1}}\right) \cdot\left(1+\frac{1}{a_{2}}\right)<1.0626 .
$$

Lemma 11. It holds that $C_{k}<192.1$.

Proof. We calculate that

$$
\begin{aligned}
C_{k} & =\left(1+\frac{3}{I_{1}}\right) I_{1}^{3} \cdot \prod_{i=1}^{k-1}\left(1+\frac{5}{I_{i+1}}\right) \cdot \frac{I_{i+1}^{3}}{I_{i}^{3}-I_{i+1}^{3}} \\
& =\left(1+\frac{3}{I_{1}}\right) I_{k}^{3} \cdot \prod_{i=1}^{k-1} \frac{1}{1-\left(I_{i+1} / I_{i}\right)^{3}}\left(1+\frac{5}{I_{i+1}}\right) .
\end{aligned}
$$

Now we distinguish two cases: If $I_{1}=16$ then $k=2$ and the last product has only one factor. Calculations show that then $\left(1+\frac{3}{I_{1}}\right) \cdot \frac{1}{1-\left(I_{2} / I_{1}\right)^{3}}\left(1+\frac{5}{I_{2}}\right)<2.72$. If $I_{1}>16$ then in particular $I_{1} \geq 2^{16}$. It can be shown that $\prod_{i=1}^{k-1} \frac{1}{1-\left(I_{i+1} / I_{i}\right)^{3}}\left(1+\frac{5}{I_{i+1}}\right)<3.01$ (similar proof as for the bound of $D_{k}$ in Lemma 10). Since $I_{1} \geq 2^{16}$ we still have that $\left(1+\frac{3}{I_{1}}\right) \prod_{i=1}^{k-1} \frac{1}{1-\left(I_{i+1} / I_{i}\right)^{3}}\left(1+\frac{5}{I_{i+1}}\right)<3.01$. Since $I_{k}^{3}=64$ in both cases we obtain the stated bound.

Note that if $I_{0} \leq 64$ then by definition $S_{0}=S_{k}$ and also $D_{k}=D_{0}=D<$ $1.0626(D+C)$ and $C_{k}=C_{0}=C<192.1$. 
4.1. Framework. Having established the existence of the schedule $S_{k}$ with the above properties we introduce our framework. The idea is the following: We split the schedule $S_{k}$ into intervals of length $I_{k}^{3}=64$. We treat each of these intervals individually as a subinstance. Let $F$ be such an interval. At the beginning of $F$ we assign each packet a random delay from the range $\{0,1, \ldots, 63\}$. The length of the resulting schedule is at most 127 . Let $\ell \in \mathbb{N}$. Using the Lovász Local Lemma we show that there are random delays for the packets such that in each subinterval of length $\ell$ at most $C_{b}^{\ell}$ packets use each edge with bandwidth $b$ (for a constant $C_{b}^{\ell}$ to be defined later). Denote by $S_{k+1}$ such a schedule. Each subinterval of length $\ell$ can now be treated as a subinstance of the packet routing problem with dilation $\ell$ and congestion $\bar{C}:=\max _{e}\left\{C_{b_{e}}^{\ell} / b_{e}\right\}$. Assume that we have a good bound $A^{b, \tau}(\bar{C}, \ell)$ for the maximum length of an optimal schedule for such an instance. This implies that by losing only a factor of $A^{b, \tau}(\bar{C}, \ell) / \ell$ we can turn $S_{k+1}$ into a feasible schedule. The length of $S_{k+1}$ then gives us our bound on the length of an optimal schedule for the original instance. As an application of this framework we derive bounds by setting $\ell:=\tau+1$.

First, we define the values $C_{b}^{\ell}$.

Definition 12. Let $b, \ell \in \mathbb{N}$. Consider $\lfloor 192.1 b\rfloor$ binary random variables $X_{i}$ such that $\operatorname{Pr}\left[X_{i}\right]=\frac{\ell}{64}$ and let $X:=\sum_{i=1}^{\lfloor 192.1 b\rfloor} X_{i}$. We define $C_{b}^{\ell}$ to be the minimum integer such that $\operatorname{Pr}\left[X>C_{b}^{\ell}\right] \cdot e \cdot\left\lceil\frac{1}{\ell} 127\right\rceil \cdot\lfloor 192.1 b\rfloor \cdot 64 \leq 1$. We write $\operatorname{Pr}\left(C_{b}^{\ell}\right):=$ $\operatorname{Pr}\left[X>C_{b}^{\ell}\right]$.

Later we will split the whole time axis into intervals of length 127 . We will split those again into even smaller intervals of length $\ell$ (or less if $\ell$ does not divide 127). To simplify notation we introduce the notion of an $\ell$-partition.

Definition 13. An $\ell$-partition of an interval $J$ with $|J|=127 \cdot M$ (for an integer $M)$ is a partition into $\left\lfloor\frac{127}{\ell}\right\rfloor \cdot M$ subintervals of length $\ell$ and $M$ subintervals of length $(k \bmod \ell)$. In the sequel we call those subintervals $\ell$-subintervals.

Using the Lovász Local Lemma, in the next lemma we show that there are random delays which turn $S_{k}$ into the schedule $S_{k+1}$ which is "almost feasible".

Lemma 14. Let $\ell, b \in \mathbb{N}$. Assume we are given an infeasible schedule $S_{k}$ of length $D_{k}$ such that in every interval of length 64 each edge $e$ is used by at most $\lfloor 192.1 b\rfloor$ packets. Then there is an infeasible schedule $S_{k+1}$ whose length is bounded by $D_{k+1}:=2 D_{k}$ that can be $\ell$-partitioned such that in every $\ell$-subinterval at most $C_{b_{e}}^{\ell}$ packets use each edge e.

Proof. We split the timeline into intervals of length $I_{k}^{3}=64$. We consider each of these intervals separately and refine them. Consider one time interval $F$ of length 64. At the beginning of $F$ we perform a random experiment and assign each packet a delay chosen uniformly and independently at random from the set $\{0,1, \ldots, 63\}$.

We define a bad event for each edge $e$. The bad event is that during an interval $[i \cdot \ell,(i+1) \cdot \ell-1] \cap F$ for some $i$ more than $C_{b_{e}}^{\ell}$ packets traverse the edge $e$. The interval $F$ was enlarged to a length of at most 127 . Hence, there are at most $\left\lceil\frac{1}{\ell} 127\right\rceil$ intervals of the form $[i \cdot \ell,(i+1) \cdot \ell-1] \cap F$. Also, the probability for each bad event is bounded by $\operatorname{Pr}\left(C_{b_{e}}^{\ell}\right) \cdot\left\lceil\frac{1}{\ell} 127\right\rceil$. Each bad event is independent from all other but at most $C_{k} b_{e} \cdot 64-1$ other bad events (follows from the bound on the congestion and the dilation). The definition of $C_{b_{e}}^{\ell}$ implies that $\operatorname{Pr}\left(C_{b_{e}}^{\ell}\right) \cdot e \cdot\left\lceil\frac{1}{\ell} 127\right\rceil \cdot\left(C_{k} b_{e} \cdot 64-1+1\right)<$ 


\begin{tabular}{|c|c|c|c|c|}
\hline \multicolumn{5}{|c|}{ Values for $\tilde{C}_{b}^{\ell}$} \\
\hline & $\ell=2$ & $\ell=3$ & $\ell=6$ & $\ell=11$ \\
\hline \hline$b=1$ & 23.6 & 29.3 & 44.3 & 66.3 \\
\hline$b=2$ & 35.8 & 45.8 & 72.8 & 113.0 \\
\hline$b=5$ & 66.4 & 87.9 & 147.3 & 239.4 \\
\hline$b=10$ & 110.5 & 150.0 & 261.2 & 435.9 \\
\hline
\end{tabular}

TABLE 2. The values $\tilde{C}_{b}^{\ell}$ for several values of $b$ and $\tau$. (Note that $\tilde{C}_{b}^{\ell}$ is an upper bound for $C_{b}^{\ell}$.)

1. The Lovász Local Lemma implies that with non-zero probability no bad event occurs. Doing this reasoning for each interval $F$ proves the existence of the schedule $S_{k+1}$ with the desired properties.

We can turn $S_{k+1}$ into a feasible schedule by solving each subinstance induced by a $\ell$-subinterval optimally. This increases the length of $S_{k+1}$ at most by a factor of (roughly) $A(\bar{C}, \ell) / \ell$ with $\bar{C}=\max _{e}\left\{\left\lceil C_{b_{e}}^{\ell} / b_{e}\right\rceil\right\}$. If we have a good bound for $A(\bar{C}, \ell)$ this yields a good bound for the length of an optimal schedule for the original instance.

In order to bound $A^{b, \tau}(\bar{C}, \ell)$ we need to estimate $\bar{C}$. As a first step, in the next lemma we upper-bound $C_{b}^{\ell}$ by a value $\tilde{C}_{b}^{\ell}$ that we will work with later.

Lemma 15. Let $b, \ell \in \mathbb{N}$. Then $C_{b}^{\ell} \leq \tilde{C}_{b}^{\ell}:=\left(1+\epsilon_{b}^{\ell}\right) \mu_{b}^{\ell}$, where $\mu_{b}^{\ell}:=\ell \cdot \frac{1}{64} \cdot 192.1 b$ and $\epsilon_{b}^{\ell}$ is the smallest real such that

$$
\left(\frac{e^{\epsilon_{b}^{\ell}}}{\left(1+\epsilon_{b}^{\ell}\right)^{1+\epsilon_{b}^{\ell}}}\right)^{\mu_{b}^{\ell}}\left\lceil\frac{1}{\ell} \cdot 127\right] \cdot e \cdot 192.1 b \cdot 64 \leq 1 .
$$

Proof. Consider the $\lfloor 192.1 b\rfloor$ binary random variables $X_{i}$ from the definition of $C_{b}^{\ell}$ and set $X:=\sum_{i=1}^{\lfloor 192.1 b\rfloor} X_{i}$. Then $\mathbb{E}[X]=\lfloor 192.1 b\rfloor \frac{\ell}{64}$. The Chernoff bounds give that

$$
p:=\operatorname{Pr}\left[X>\left(1+\epsilon_{b}^{\ell}\right) \mu_{b}^{\ell}\right] \leq \operatorname{Pr}\left[X \geq\left(1+\epsilon_{b}^{\ell}\right) \mu_{b}^{\ell}\right] \leq\left(\frac{e^{\epsilon_{b}^{\ell}}}{\left(1+\epsilon_{b}^{\ell}\right)^{1+\epsilon_{b}^{\ell}}}\right)^{\mu_{b}^{\ell}} .
$$

By the choice of $\epsilon_{b}^{\ell}$ this implies that

$$
\operatorname{Pr}\left[X \geq C_{b}^{\ell}\right] \cdot e \cdot\left\lceil\frac{1}{\ell} 127\right\rceil \cdot 192.1 b \cdot 64 \leq 1
$$

Table 2 shows bounds for $\tilde{C}_{b}^{\ell}$ for some values $b$ and $\ell$. We note that for these values for fixed $\ell$ and increasing $b$ the values $\tilde{C}_{b}^{\ell} / b$ do not increase. We show in the following lemma that this holds in general. This will become useful later: it allows us to argue that $\bar{C}=\max _{e}\left\{\left\lceil C_{b_{e}}^{\ell} / b_{e}\right\rceil\right\} \leq \max _{e}\left\{\left\lceil\tilde{C}_{b_{e}}^{\ell} / b_{e}\right\rceil\right\}=\tilde{C}_{b}^{\ell} / b$, where $b$ denotes the minimum bandwidth of any edge in the instance.

Lemma 16. Let $b, b^{\prime}, \ell \in \mathbb{N}$ with $b \leq b^{\prime}$. Then $\tilde{C}_{b}^{\ell} / b \geq \tilde{C}_{b^{\prime}}^{\ell} / b^{\prime}$. 
Proof. It suffices to show the claim for $b^{\prime}:=b+1$. By definition, $\tilde{C}_{b}^{\ell}=\left(1+\epsilon_{b}^{\ell}\right) \cdot \frac{\ell}{64}$. $192.1 b$ and $\tilde{C}_{b+1}^{\ell}=\left(1+\epsilon_{b+1}^{\ell}\right) \frac{\ell}{64} \cdot 192.1(b+1)$ with $\epsilon_{b}^{\ell}$ and $\epsilon_{b+1}^{\ell}$ as defined in Lemma 15. Hence, it suffices to show that $\epsilon_{b+1}^{\ell} \leq \epsilon_{b}^{\ell}$. By definition, we have that

$$
192.1 b \cdot 64 \cdot\left\lceil\frac{1}{\ell} \cdot 127\right\rceil \cdot e \cdot\left(\frac{e^{\epsilon_{b}^{\ell}}}{\left(1+\epsilon_{b}^{\ell}\right)^{1+\epsilon_{b}^{\ell}}}\right)^{\frac{\ell}{64} \cdot 192.1 b} \leq 1 .
$$

To show that $\epsilon_{b+1}^{\ell} \leq \epsilon_{b}^{\ell}$ we calculate that

$$
\begin{aligned}
& 192.1(b+1) \cdot 64 \cdot\left\lceil\frac{1}{\ell} \cdot 127\right] \cdot e\left(\frac{e^{\epsilon_{b}^{\ell}}}{\left(1+\epsilon_{b}^{\ell}\right)^{1+\epsilon_{b}^{\ell}}}\right)^{\frac{\ell}{64} \cdot 192 \cdot 1(b+1)} \\
= & 192.1 b \cdot 64\left[\frac{1}{\ell} \cdot 127\right] \cdot e \cdot\left(\frac{e^{\epsilon_{b}^{\ell}}}{\left(1+\epsilon_{b}^{\ell}\right)^{1+\epsilon_{b}^{\ell}}}\right)^{\frac{\ell}{64} \cdot 192.1 b} \frac{(b+1)}{b}\left(\frac{e^{\epsilon_{b}^{\ell}}}{\left(1+\epsilon_{b}^{\ell}\right)^{1+\epsilon_{b}^{\ell}}}\right)^{\frac{\ell}{64} 192.1} .
\end{aligned}
$$

Hence, it remains to show that

$$
\frac{b+1}{b}\left(\frac{e^{\epsilon_{b}^{\ell}}}{\left(1+\epsilon_{b}^{\ell}\right)^{1+\epsilon_{b}^{\ell}}}\right)^{\frac{\ell}{64} 192.1} \leq 1
$$

or equivalently

$$
\left(\frac{b+1}{b}\right)^{b}\left(\frac{e^{\epsilon_{b}^{\ell}}}{\left(1+\epsilon_{b}^{\ell}\right)^{1+\epsilon_{b}^{\ell}}}\right)^{\frac{\ell}{64} \cdot 192.1 b} \leq 1^{b}=1 .
$$

From Inequality 4.2 we conclude that

$$
\left(\frac{e^{\epsilon_{b}^{\ell}}}{\left(1+\epsilon_{b}^{\ell}\right)^{1+\epsilon_{b}^{\ell}}}\right)^{\frac{\ell}{64} \cdot 192.1 b} \leq \frac{1}{192.1 b \cdot 64 \cdot\left\lceil\frac{1}{\ell} \cdot 127\right\rceil \cdot e} .
$$

For proving Inequality 4.3 it hence suffices to show that

$$
\left(1+\frac{1}{b}\right)^{b} \leq 192.1 b \cdot 64 \cdot\left\lceil\frac{1}{\ell} \cdot 127\right\rceil \cdot e .
$$

We have that $\lim _{b \rightarrow \infty}\left(1+\frac{1}{b}\right)^{b}=e$. Moreover, since $192.1 b \cdot 64 \cdot\left\lceil\frac{1}{\ell} \cdot 127\right\rceil \cdot e \geq e$ for all $b$ Inequality 4.3 holds. This implies that $\epsilon_{b+1}^{\ell} \leq \epsilon_{b}^{\ell}$.

In the following lemma we formalize how we can derive bounds for general instances using having good bounds for $A^{b, \tau}(C, \ell)$ (for some value $\ell$ ).

Lemma 17. Let $I$ be an instance of the packet routing problem with minimum bandwidths and transit times $b$ and $\tau$, respectively, and let $\ell, M \in \mathbb{N}$. Assume we are given an infeasible schedule $S_{k+1}$ for I of length $127 \cdot M$ which is $\ell$-partitioned 
such that every $\ell$-subinterval is used by at most $C_{b}^{\ell}$ packets. Then there is a feasible schedule for I whose length is bounded by

$$
\left\lceil\frac{127}{\ell}\right\rceil \cdot M \cdot A^{b, \tau}\left(\max _{e}\left\{\left\lceil C_{b_{e}}^{\ell} / b_{e}\right\rceil\right\}, \ell\right) .
$$

Proof. We change the given schedule $S_{k+1}$ to a feasible schedule by refining each $\ell$-subinterval of the $\ell$-partition. Each $\ell$-subinterval can be modeled as an instance of the packet routing problem. This subinstance has a dilation of at most $\ell$ and each edge $e$ is used by at most $C_{b_{e}}^{\ell}$ packets. Hence, the congestion of this subinstance is $\bar{C}:=\max _{e}\left\{\left\lceil C_{b_{e}}^{\ell} / b_{e}\right\rceil\right\}$. According to our assumption concerning $b$ and $\tau$ the schedule in each $\ell$-subinterval can be refined to a feasible schedule of length $A^{b, \tau}\left(\max _{e}\left\{\left\lceil C_{b_{e}}^{\ell} / b_{e}\right\rceil\right\}, \ell\right)$. This yields the bound stated in the theorem.

Later, we will work with the values $\tilde{C}_{b}^{\ell}$ instead of the values $C_{b}^{\ell}$. For giving a general theorem for the bounds derived by our framework, we study the value $\tilde{C}_{b}^{\ell} / b$.

Lemma 18. It holds that

$$
\left\lceil\tilde{C}_{b}^{\ell} / b\right\rceil \leq 3.01 \ell \cdot g(\ell, b)
$$

for a function $g(\ell, b)$ which approaches 1 for increasing $\ell$ or $b$.

Proof. From Lemma 15 we derive that $\tilde{C}_{b}^{\ell}:=\left(1+\epsilon_{b}^{\ell}\right) \mu_{b}^{\ell}$ where $\mu_{b}^{\ell}:=\ell \cdot \frac{1}{64} \cdot 192.1 b$ and $\epsilon_{b}^{\ell}$ is the smallest real such that

$$
\left(\frac{e^{\epsilon_{b}^{\ell}}}{\left(1+\epsilon_{b}^{\ell}\right)^{1+\epsilon_{b}^{\ell}}}\right)^{\mu_{b}^{\ell}}\left\lceil\frac{1}{\ell} \cdot 127\right\rceil \cdot e \cdot 192.1 b \cdot 64 \leq 1 .
$$

We see that for fixed $\ell$ and increasing $b$, the value $\epsilon_{b}^{\ell}$ decreases. Similarly, for fixed $b$ and increasing $\ell$, we also have that $\epsilon_{b}^{\ell}$ decreases. We conclude that $\tilde{C}_{b}^{\ell}$ approaches $\mu_{b}^{\ell}=\ell \cdot \frac{1}{64} \cdot 192.1 b \leq 3.01 \ell \cdot b$ for increasing $\ell$ or increasing $b$.

Now we can prove our main theorem for the bounds derived by our framework.

Theorem 19. Let $I$ be an instance of the packet routing problem with minimum bandwidths and transit times $b$ and $\tau$, respectively. For any $\ell \in \mathbb{N}$ there is a feasible schedule for I whose length is bounded by

$$
A^{b, \tau}(3.01 \ell \cdot f(\ell, b), \ell) \cdot\left(\frac{2.11}{\ell}+\delta\right)(C+D)
$$

for a function $f(\ell, b)$ which tends to 1 for increasing $\ell$ or $b$ and with $\delta \leq \frac{1}{60}$.

Proof. We have that $C_{b}^{\ell} \leq \tilde{C}_{b}^{\ell}$ for all $\ell$ and $b$. From Lemma 16 it follows that $\max _{e}\left\{\left\lceil\tilde{C}_{b_{e}}^{\ell} / b_{e}\right\rceil\right\} \leq\left\lceil\tilde{C}_{b}^{\ell} / b\right\rceil$. With Lemma 17 and 18 we obtain a bound of

$$
\begin{aligned}
& A^{b, \tau}\left(\left\lceil\tilde{C}_{b}^{\ell} / b\right\rceil, \ell\right) \cdot\left\lceil\frac{127}{\ell}\right\rceil \cdot \frac{D_{k}}{64} \\
< & A^{b, \tau}(3.01 \ell \cdot f(\ell, b), \ell) \cdot\left\lceil\frac{127}{\ell}\right\rceil \cdot \frac{1.0626(C+D)}{64}
\end{aligned}
$$

Note that here we used that $(C+D)$ is large as mentioned in the introduction since then $\left\lceil\frac{D_{k}}{64}\right\rceil \approx \frac{D_{k}}{64}$. Since $D_{k}$ is strictly smaller than $1.0626(D+C)$ there is a value 
$N_{0}$ such that for all $C, D$ with $(C+D) \geq N_{0}$ our bounds hold. To simplify the expression further we calculate that

$$
\frac{1}{64} \cdot\left\lceil\frac{127}{\ell}\right\rceil \cdot 1.0626 \leq \frac{1.0626}{64} \cdot \frac{127+\ell}{\ell} \leq \frac{2.11}{\ell}+\frac{1}{60} .
$$

Theorem 2 allows us to bound the expression $A^{b, \tau}(C, \ell)$ for the case that $\ell=$ $\tau+1$. Using this insight we can use the theorem above to derive general bounds for all packet routing instances.

Theorem 20. Let I be an instance of the packet routing problem with minimum bandwidths and transit times $b$ and $\tau$, respectively. There is a feasible schedule for $I$ whose length is bounded by

$$
\left(6.4 \cdot f(\tau+1, b)+2.11 \frac{\tau}{\tau+1}+\delta\right)(C+D) \leq(8.51 \cdot f(\tau+1, b)+\delta)(C+D)
$$

for a function $f(\tau+1, b)$ which tends to 1 for increasing $\tau$ or $b$ and with $\delta \leq$ $\frac{1}{60}(\tau+3.01 \cdot f(\tau+1, b)(\tau+1))$.

Proof. We choose $\ell:=\tau+1$ in Theorem 19. Theorem 2 shows that $A^{b, \tau}(C, \tau+1) \leq$ $C+\tau$. This gives a bound of

$$
(3.01(\tau+1) \cdot f(\tau+1, b)+\tau) \cdot\left(\frac{2.11}{\tau+1}+\delta^{\prime}\right)(C+D)
$$

for $\delta^{\prime}=\frac{1}{60}$. Calculations show that this expression is upper-bounded by the expression stated in the theorem.

Note here that - for values of $f(\tau+1, b)$ close to 1 - the formula stated in Theorem 20 gives better bounds if $\tau=1$ than for higher values of $\tau$. However, for small $\tau$ and $b$ the bound of the formula improves as $\tau$ increases.

Observe that $\delta$ increases for increasing $\tau$. This worsens the bound for very large values $\tau$. However, in Section 4.2 we prove a much better bound for the case that $\tau \geq 63$.

4.2. High values for $\tau$. Given the schedule $S_{k}$, in our framework we used the Lovász Local Lemma to prove the existence of the schedule $S_{k+1}$. However, we can alternatively turn the schedule $S_{k}$ to a feasible schedule directly. This is in particular useful for cases where $\tau$ is relatively large, as we will see in Corollary 22.

Theorem 21. Let $I$ be an instance of the packet routing problem with minimum bandwidth $b$ and minimum transit time $\tau$. There is feasible schedule for I whose length is bounded by

$$
\frac{1}{60}(C+D) \cdot A^{b, \tau}(193,64) .
$$

Proof. Recall that we proved the existence of the schedule $S_{k}$ which has the property that in every interval of length 64 each edge is used by at most $C_{k} b=192.1 b$ packets. Hence, there is a feasible schedule for $I$ whose length is bounded by

$$
\frac{D_{k}}{64} \cdot A^{b, \tau}\left(\left\lceil C_{k}\right\rceil, 64\right)<\frac{1.0626(D+C)}{64} \cdot A^{b, \tau}(193,64)
$$


which is bounded by the expression above. Note that here again we used that $(C+D)$ is large as mentioned in the introduction since then $\left\lceil\frac{D_{k}}{64}\right\rceil \approx \frac{D_{k}}{64}$. Since $D_{k}$ is strictly smaller than $1.0626(D+C)$ there is a value $N_{0}$ such that for all $C, D$ with $(C+D) \geq N_{0}$ our bounds hold.

Using our insight gained in Theorem 2 for $A^{b, \tau}\left(C_{k}, \tau+1\right)$ allows us to prove the following Corollary.

Corollary 22. Let I be an instance of the packet routing problem with minimum bandwidth $b$ and minimum transit time $\tau \geq 63$. Then there is always a packet routing schedule whose length is bounded by $4.25(C+D)$.

Proof. For $\tau \geq 63$ we have that $64 \leq \tau+1$. Hence, Theorem 2 implies that $A^{b, \tau}(193,64) \leq 193+64-1=255$ if $\tau \geq 63$. Plugging this into the bound of Theorem 21 yields the bound of $4.25(C+D)$.

Table 1 shows some bounds for the lengths of schedules depending on $\tau$ and $b$.

4.3. Unit Transit Times and Unit Bandwidths. Finally, we use the above framework to derive a bound of $23.4(C+D)$ for the case of unit transit times and unit bandwidths. This improves the bound of $39(C+D)$ proven by Scheideler [22]. First, we precisely calculate $C_{1}^{2}$ (instead of using the estimation $\tilde{C}_{1}^{2}$ ).

Lemma 23. It holds that $C_{1}^{2}=21$.

Proof. Since the value $C_{1}^{2}$ does not depend on any parameters it can be calculated exactly. Calculations using e.g., MATLAB then give the desired result.

Now we can use our framework together with the above lemma to derive our desired bound.

Theorem 24. Let $I$ be an instance of the packet routing problem with $b=1$ and $\tau=1$. Then there is a feasible schedule for $S$ whose length is bounded by $23.4(C+D)$.

Proof. Let $\ell:=2$. If all edges have unit transit times and bandwidths then $\max _{e}\left\{\left[C_{b_{e}}^{\ell} / b_{e}\right\rceil\right\}=C_{1}^{2}$. Hence, in the reasonings of Lemma 19 and Theorem 20 we can use $C_{1}^{2}$ instead of $\tilde{C}_{1}^{2}$. This gives a bound of $\left(C_{1}^{2}+1\right) \cdot 1.0626(C+D)$. Using that $C_{1}^{2}=21$ as derived in Lemma 23 gives the stated bound.

Acknowledgements. We would like to thank Jan-Philipp Kappmeier, Olaf Maurer, and Jannik Matuschke for fruitful discussions and in particular Jan-Philipp Kappmeier for helpful comments on the draft.

\section{REFERENCES}

[1] M. Adler, S. Khanna, R. Rajaraman, and A. Rosén. Time-constrained scheduling of weighted packets on trees and meshes. Algorithmica, 36:123-152, 2003.

[2] M. Adler, R. Sitaraman, A. Rosenberg, and W. Unger. Scheduling time-constrained communication in linear networks. In Proceedings of the 10th annual ACM symposium on Parallel Algorithms and Architectures (SPAA 1998), pages 269-278, 1998.

[3] C. Busch, M. Magdon-Ismail, M. Mavronicolas, and P. Spirakis. Direct routing: Algorithms and complexity. Algorithmica, 45:45-68, 2006.

[4] M. di Ianni. Efficient delay routing. Theoretical Computer Science, 196:131-151, 1998.

[5] L. Fleischer and M. Skutella. Minimum cost flows over time without intermediate storage. In Proceedings of the 14th Annual Symposium on Discrete Algorithms (SODA 2003), 2003. 
[6] L. Fleischer and M. Skutella. Quickest flows over time. SIAM Journal on Computing, 36:16001630, 2007.

[7] A. Hall, S. Hippler, and M. Skutella. Multicommodity flows over time: Efficient algorithms and complexity. Theoretical Computer Science, 2719:397-409, 2003.

[8] A. Hall, K. Langkau, and M. Skutella. An FPTAS for quickest multicommodity flows with inflow-dependent transit times. Algorithmica, 47:299-321, 2007.

[9] F. Meyer Auf Der Heide and B. Vocking. Shortest paths routing in arbitrary networks. Journal of Algorithms, 31:105-131, 1999.

[10] B. Hoppe and É. Tardos. The quickest transshipment problem. Mathematics of Operations Research, 25:36-62, 2000.

[11] R. Koch, B. Peis, M. Skutella, and A. Wiese. Real-time message routing and scheduling. In S. Naor, editor, Proceedings of the 12th International Workshop on Approximation Algorithms for Combinatorial Optimization Problems (APPROX 2009), volume 5687 of LNCS, pages 217-230, 2009

[12] F. T. Leighton, B. M. Maggs, and S. B. Rao. Packet routing and job-scheduling in O(congestion + dilation $)$ steps. Combinatorica, 14:167-186, 1994.

[13] F. T. Leighton, B. M. Maggs, and A. W. Richa. Fast algorithms for finding $O$ (congestion + dilation) packet routing schedules. Combinatorica, 19:375-401, 1999.

[14] F. T. Leighton, F. Makedon, and I. G. Tollis. A $2 n-2$ step algorithm for routing in an $n \times n$ array with constant size queues. In Proceedings of the 1st Annual Symposium on Parallel Algorithms and Architectures (SPAA 1989), pages 328-335, 1989.

[15] J. Y.-T. Leung. Handbook of Scheduling: Algorithms, Models and Performance Analysis. CRC Press, Inc., 2004.

[16] Y. Mansour and B. Patt-Shamir. Many-to-one packet routing on grids. In Proceedings of the 27th Annual Symposium on Theory of Computing (STOC 1995), pages 258-267, 1995.

[17] R. Ostrovsky and Y. Rabani. Universal $O\left(\right.$ congestion + dilation $\left.+\log ^{1+\epsilon} N\right)$ local control packet switching algorithms. In Proceedings of the 29th annual ACM Symposium on Theory of Computing (STOC 1997)pack, pages 644-653, 1997.

[18] B. Peis, M. Skutella, and A. Wiese. Packet routing: Complexity and algorithms. In Proceedings of the 7th Workshop on Approximation and Online Algorithms (WAOA 2009), volume 5893 of LNCS, pages 217-228. Springer, 2010.

[19] B. Peis, M. Skutella, and A. Wiese. Packet routing on the grid. In Proceedings of the 9th Latin American Theoretical Informatics Symposium (LATIN 2010), volume 6034 of LNCS, pages 120-130. Springer, 2010.

[20] Y. Rabani and É. Tardos. Distributed packet switching in arbitrary networks. In Proceedings of the 28th annual ACM Symposium on Theory of Computing (STOC 1996), pages 366-375. ACM, 1996.

[21] S. Rajasekaran. Randomized algorithms for packet routing on the mesh. Technical Report MSCIS-91-92, Dept. of Computer and Information Sciences, Univ. of Pennsylvania, Philadelphia, PA, 1991.

[22] C. Scheideler. Offline routing protocols. Universal Routing Strategies for Interconnection Networks, LNCS, 1390:57-71, 1998.

[23] A. Srinivasan and C.-P. Teo. A constant-factor approximation algorithm for packet routing and balancing local vs. global criteria. SIAM Journal on Computing, 30:636-643, 2001. 